\title{
Light and heavy multiquark spectroscopy ${ }^{* \dagger}$
}

\author{
Jean-Marc Richard ${ }^{\text {a }}$ \\ ${ }^{a}$ Laboratoire de Physique Subatomique et Cosmologie, \\ Université Joseph Fourier-IN2P3-CNRS, \\ 53, avenue des Martyrs, 38026 Grenoble cedex, France
}

The dynamics of multiquark binding is revisited in the light of the recent experimental results. It is emphasized that some configurations mixing light and heavy flavours are among the most favourable for stable or metastable multiquarks. The nuclear-physics type of approach predicting the so-called hadron-hadron molecules is compared to direct studies in terms of quark interaction.

\section{Introduction}

The recently found or claimed new hadrons aroused a renewed interest into the spectroscopy of rare or exotic particles 12. The activity in this field is unfortunately rather fluctuating. For instance, experiments having ample data sets with pions, kaons, protons and neutrons did not look very seriously at invariant mass plots with baryon number $B=1$ and strangeness $S=1$, until some pentaquark event was reported elsewhere. Similarly, the theoretical searches in the sector of exotic hadrons follow the fashion. Shortly after the announcement of the pentaquark peaks, several estimates of multiquark masses and properties have been published, which, however interesting, could have benefited from more extensive training.

An exception is the class of calculations based on potential models, where the know-how of fewbody quantum mechanics is used to appreciate the difference between mere scattering states and possible resonances. See, e.g., Hiyama et al. [3]. However, these estimates are based on a somewhat ad-hoc extension of the quark-antiquark potential to situations involving more quarks and antiquarks. It would be desirable to combine this approach with better dynamical ingredients.

\footnotetext{
${ }^{*}$ Invited Talk at QCD05, 12th International QCD Conference, July 4-9, 2005, Montpellier (France), to appear in the Proceedings, ed. S. Narison

${ }^{\dagger}$ Preprint lpsc-05-121, ArXiv:hep-ph/0601043

$\ddagger$ email: jean-marc.richard@lpsc.in2p3.fr
}

\section{Salient experimental results}

In recent months, several new hadrons have been claimed or firmly established, among them: low-lying scalar and axial mesons with charm and strangeness $C=S=1$, i.e., flavour content $(c \bar{s})$, the so-called $\mathrm{D}_{s, J}^{*}$; double-charm baryons, $(c c q)$, seen by the SELEX experiment; controversial candidates for light and heavy pentaquarks; discovery of the long-awaited missing states of charmonium $\eta_{c}^{\prime}$ and $h_{c}$; evidence for new meson resonances with hidden charm, $\mathrm{X}(3872), \mathrm{X}(3940)$ and $Y(4260)$; not to mention new information of light scalar mesons, excitations of singly-charmed baryons, etc.

It is worth stressing that the new findings are not always due to an increase of statistics. For instance, the singlet states of charmonium have been eventually discovered using new production and new decay channels.

As for pentaquarks, the superposition of positive and negative results remains very puzzling, especially for non-experts. See, e.g., the review by S. Kabana at this Conference [1. Data were perhaps cut off too sharply in some of the analyses leading to tentative peaks. Even a theorist can understand that a sequential production scheme would produce peaks for any pair of final-state particles, if it is restricted to collinear events. The robustness of peaks when opening the angles is a crucial test for genuine resonances. 


\section{Models}

These discoveries stimulated an intense theoretical activity. Old ideas about exotics were re-examined and confronted to other proposed mechanisms. In particular:

\subsection{Hybrids}

Years ago, it has been suggested that the quark-antiquark system ${ }^{4}$ might experience new types of excitations [4, beyond conventional radial or orbital excitations, and this idea is confirmed by QCD sum rules and lattice QCD. This is very similar to the spectroscopy of $\mathrm{H}_{2}{ }^{+}$, with a first series of "ordinary" states corresponding to the two protons moving in the lowest BornOppenheimer potential, and "exotic states" occurring when the electron is excited. It is extremely plausible that at least one of the $\mathrm{X}(3872)$, $\mathrm{X}(3940)$ and $\mathrm{Y}(4260)$ states is an hidden-charm hybrid $(c \bar{c} g)$. The selection rules specific to the decay and production of hybrids have to be checked to confirm this hypothesis [5].

\subsection{Chiral dynamics}

Light-quark dynamics predicts parity partners for high-lying excitations of hadrons, a pattern which is seemingly observed [2], and also parity partners of ground states with favourable quantum numbers. See, e.g., Ref. 67] for a discussion and further references. If the $\mathrm{D}_{s, J}^{*}$ are chiral partners of the ground-state $\left\{\mathrm{D}_{s}, \mathrm{D}_{s}^{*}\right\}$, the question is whether this is a more realistic picture of the $0^{+}$and $1^{+}$members of the $(c \bar{s})$ sector, or supernumerary states, with ordinary orbital excitations awaiting identification. This latter scenario would be reminiscent of the situation which is observed for light scalar mesons, with too many states as compared to a naive quark-model counting.

\subsection{Yukawa dynamics}

Some pioneers made the observation that nuclear forces are by no means restricted to the nucleon-nucleon system (for refs., see, e.g., 8 8 . and the review by Swanson [2]). For any pair of hadrons containing light quarks, a similar interaction should be present, and might well produce

${ }^{4}$ Hybrids baryons are also predicted bound states, if the long-range pion-exchange is allowed and turns out to be attractive ${ }^{5}$. Note, however, the warning by Suzuki, that the massdifference between $\mathrm{D}$ and $\mathrm{D}^{*}$ suppresses the effectiveness of the potential induced by pion exchange [9].

\subsection{Borromean binding}

If you remain sceptical, arguing that the meson-meson attraction is presumably too weak to achieve the binding of two mesons ${ }^{6}$, you should not eliminate definitely the possibility of hadron molecules with charm. The phenomenon of "Borromean" binding, more familiar in nuclear and molecular physics, tells us that a strength about $20 \%$ too weak for 2-body binding is sufficient for 3 -body binding. Hence a $\left[\mathrm{DD}^{*} \overline{\mathrm{D}}\right]$ state might exist, with the star circulating from one constituent to the other, following the exchanged pions between them. This idea of Borromean molecules was applied to hadrons by Bicudo in the context of pentaquark, and further studied by others. In this picture, the light pentaquark, for instance, is seen as a $(\mathrm{N}, \mathrm{K}, \pi)$ compound, whose none of the 2-body subsystems is stable. See, Ref. [11.

\subsection{Diquark chemistry}

The success of the quark model relies on the complicated dressing of quarks by gluons reducing approximately to massive quarks interacting through a confining potential. A further simplification consists of regrouping two quarks in a baryon to form a diquark, which, in turn, forms a quarkonium-like structure with the third quark. This leads to a successful phenomenology of baryon spectroscopy, baryon production in diffractive processes, etc.

In the good old time of baryonium, where all dreams were permitted, a colour $\overline{3}$ or 6 diquark was imagined to rotate around its colourconjugate antidiquark, to form new types of me-

\footnotetext{
${ }^{5}$ For non-experts, it suffices to stress that the one-pionexchange potential depends on spin and isospin, and does not operate on pseudoscalar particles. For DD*, D flips to $\mathrm{D}^{*}$ and vice-versa each times a pion is emitted or absorbed. ${ }^{6}$ In our valley of tears with $d=3$ space dimensions, a short-range attraction call for a minimal strength to produce a quantum bound state, unlike the more favourable cases with $d=1$ or $d=2$.
} 
son structures. It was not clearly demonstrated, however, how this clustering occurs in a $(q q \bar{q} \bar{q})$ system when orbital momentum is implemented. The question remained unsolved, and even untouched, as baryonium disappeared from the tables. In contrast, Martin [12] demonstrated that in a large class of models, high- $\ell$ baryons have a $[(q q)-q]$ structure that was postulated to explain the slope of Regge trajectories.

The concept of diquark has been recently revisited and applied to supernumerary scalar mesons, pentaquark states and hidden-charm mesons that cannot be too easily accommodated as mere $(c \bar{c})$ levels [13. A triquark $(u d \bar{s})$ was even proposed 14. This gives an appealing and unified picture of these hadrons. If the quark dynamics is such that three quarks never form a $(q q q)$ structure to leave the scene to diquarks, three diquarks or three triquarks would presumably never combine together. Hence, structures like $(q q)^{3}$, or $(u d \bar{s})^{3}$ are avoided, which otherwise would be somewhat embarrassing predictions. Diquarks are not frozen for ever, they are effective entities at work in a given context. Hence models based on diquarks should not be extrapolated without care.

Recently Maiani et al. described the new hidden-charm resonances in terms of diquarks $(c q)$ or $(c s)$. See, e.g., [15] and refs. there.

\subsection{Chromomagnetic binding}

The hyperfine splitting of ground-state hadrons, such as $\Delta-\mathrm{N}$ or $\mathrm{J} / \psi-\eta_{c}$ is well described by a chromomagnetic term $-\sum_{i<j} C_{i j} \vec{\sigma}_{i} \cdot \vec{\sigma}_{j} \tilde{\lambda}_{i} \cdot \tilde{\lambda}_{j}$, inspired from one-gluon exchange, but covering a wider range of microscopic mechanisms.

It was stressed that this Hamiltonian might lead to coherent attraction, i.e., assume in certain multiquark configurations a value which is larger (in absolute value) that the sum of its contributions to the hadrons in the threshold, hence favouring the stability of this multiquark against spontaneous dissociation. This concerns in particular the dibaryon $\mathrm{H}(u$ uddss) [16 or the 1987vintage pentaquark $\mathrm{P}(\bar{c} u u d s)$ (or these obtained by permuting $u, d$ and $s$ ) 17. The $\mathrm{H}$ was desperately searched for, and the $\mathrm{P}$ moderately, without success. This was explained by unfavourable ef- fects of flavour-symmetry or weaker short-range correlation in multiquarks. More extreme are the heretic attempts to substitute to the above chromomagnetic Hamiltonian terms inspired by instanton interaction, or spin-flavour effects.

The wave of exoticism led Høgaasen et al. to revisit the chromomagnetic Hamiltonian. When flavour symmetry is properly taken into account, the results appear somewhat at variance with respect to the earlier estimates. For instance, light scalar mesons with hidden strangeness are pushed up in the spectrum, and the eigenstates with mass of about $1 \mathrm{GeV}$ have little $\mathrm{K} \overline{\mathrm{K}}$ content. In the $(c \bar{c} q \bar{q})$, a remarkable eigenstate appears which almost miraculously exhibits the mass and coupling pattern of the $X(3872)$.

\subsection{Chromoelectric binding}

Remember the (almost) local writer: "Il ferma la porte à double tour. Malheureusement, il avait oublié la fenêtre ${ }^{7 "}$, which can be translated here as: If none of the previous scenarios for exotic hadrons has succeeded, do not give up, yet. Why not try something simple and straightforward if the most adventurous speculations have failed. A nice feature of QCD is flavour independence. For instance, the same potential achieves a good picture of both charmonium and bottomonium families and gives predictions for $(b \bar{c})$. The same situation is observed in atomic physics with the same Coulomb potential acting for electrons, muons and antiprotons. It is noticed here that while the equal-mass positronium molecule $\left(\mathrm{e}^{+}, \mathrm{e}^{+}, \mathrm{e}^{-}, \mathrm{e}^{-}\right)$ is weakly bound, the unequal mass configuration $\left(\mathrm{p}, \mathrm{p}, \mathrm{e}^{-}, \mathrm{e}^{-}\right)$is deeply bound. This suggests that exotic mesons with two units of heavy flavour, that is to say, $(Q Q \bar{q} \bar{q})$, might exist below the threshold for spontaneous dissociation and hence be rather narrow. This possibility, proposed years ago, has received further theoretical support [18].

\section{Outlook}

Hopefully the activity developed after the announcement of the pentaquark will survive its disappearance. Understanding the origin of quark

\footnotetext{
${ }^{7}$ Alphonse Daudet, in La chèvre de Monsieur Seguin, part
} of "Les Lettres de mon Moulin". 
confinement is, indeed, a fascinating program. Once models are built or basic QCD calculations are performed to account for the main properties of quark-antiquark and three-quarks systems, it is tempting to examine higher configurations, or molecules made of simple hadrons.

It often happens that states containing both heavy and light quarks and antiquarks offer the best chances for multiquark binding. Hence these flavour states should be preferentially looked at. There are already indications of unusual hadron states in the hidden-charm sector. The double charm sector is also accessible, as demonstrated by the detection of double-charm baryons at SELEX and double-charmonium production at $\mathrm{B}$ factories.

A debate cannot be avoided between the models based on long-range hadron dynamics and those based mostly on direct quark interaction. In nuclei, the Yukawa interaction remains at work because the short-range repulsion (and the Fermi statistics) prevents the nucleons to merge into a single bag. In absence of evidence for such a short-range repulsion applicable to $\mathrm{DD}^{*}$, it is not sure that the long-range interaction plays a leading role. This was already a problem with the so-called quasi-nuclear of baryonium. Shapiro, Dover and others predicted several interesting $N \bar{N}$ bound states and resonances, but never elucidated satisfactorily how they survive short-range effects. On the other hand, the vicinity of many states to their major decay threshold is a clear signal that the hadron-hadron physics is present there.

I would like to thank S. Narison for this beautiful conference, and R.D. Matheus for stimulating discussions. Due to the lack of space, it is impossible to quote here all the interesting contributions to this field, and I apologize for the omissions.

\section{REFERENCES}

1. The experimental results are presented in several talks given at this Conference.

2. E. Klempt, Hadron05 summary: Experiment; T. Barnes. Hadron05 summary: Heavy quark hadrons and theory, arXiv:hep-ph/0510365
Eric Swanson. Hadron05: Review of heavy hadron spectroscopy; Shan Jin, Int. J. Mod. Phys., A20:5145-5155, 2005.

3. E. Hiyama et al. Phys. Lett. B (in press), 2005 arXiv:hep-ph/0507105.

4. R. Giles, S.H.H. Tye. Phys. Rev., D16:1079, 1977; D. Horn, J. Mandula. Phys. Rev., D17:898, 1978; P. Hasenfratz, R.R. Horgan, J. Kuti, J. M. Richard, Phys. Lett., B95:299, 1980.

5. F.E. Close, P.R. Page, Phys. Lett., B628:215222, 2005; E. Kou, O. Pene. Phys. Lett., B631:164-169, 2005.

6. L.Ya. Glozman, A.V. Nefediev, J.E.F.T. Ribeiro, Phys. Rev., D72:094002, 2005.

7. W.A. Bardeen, E.J. Eichten, C.T. Hill. Phys. Rev., D68:054024, 2003."

8. N.A. Törnqvist, $3872-\mathrm{MeV}$ as a Phys. Lett., B590:209-215, 2004; M. B. Voloshin, component Phys. Lett.,B 579:316-320, 2004; E. Braaten and M. Kusunoki, 3870-MeV," Phys. Rev., D69:074005, 2004

9. M. Suzuki. Phys. Rev.,D 72:114013, 2005.

10. J. M. Richard, in V.B. Belyaev's 70th birthday book, arXiv:nucl-th/0305076

11. P. Bicudo. Phys. Rev., D71:011501, 2005; A. Hosaka et al. ibidem C71:045205, 2005.

12. A.Martin. Z. Phys., C32:359, 1986.

13. R.L. Jaffe. Phys. Rept., 409:1-45, 2005.

14. M. Karliner, H.J. Lipkin. Phys. Lett., B575:249-255, 2003.

15. L. Maiani, F. Piccinini, A. D. Polosa, V. Riquer, arXiv:hep-ph/0512082.

16. R.L. Jaffe. Phys. Rev. Lett., 38:195-198, 1977.

17. C. Gignoux, B. Silvestre-Brac, J. M. Richard. Phys. Lett., B193:323, 1987; H.J. Lipkin. Phys. Lett., B195:484, 1987.

18. B.A. Gelman, S. Nussinov. Phys. Lett., B551:296-304, 2003; D. Janc, M. Rosina. Few Body Syst., 35:175-196, 2004; J. M. Richard, F. Stancu, arXiv:hep-ph/0511043 J. Vijande, F. Fernandez, A. Valcarce, B. Silvestre-Brac, Eur. Phys. J., A19: 383, 2004. 